\title{
Revisiting the Electronic Structure of Phosphazenes.
}

Adrian B. Chaplin, ${ }^{\mathrm{a} *}$ John A. Harrison, ${ }^{\mathrm{b}}$ and Paul J. Dyson ${ }^{\mathrm{a}}$

${ }^{a}$ Institut des Sciences et Ingénierie Chimiques, Ecole Polytechnique Fédérale de Lausanne (EPFL),CH-1015 Lausanne, Switzerland. E-mail: adrian.chaplin@epfl.ch ${ }^{b}$ Department of Chemistry, Institute of Fundamental Sciences, Massey University at Auckland, Private Bag 102 904, North Shore Mail Centre, Auckland, New Zealand.

\section{SUPPORTING INFORMATION}

\section{S1. Geometry optimisations}

Table S-1: Geometry optimisation of $\left[\mathrm{N}_{3} \mathrm{P}_{3} \mathrm{Cl}_{6}\right]$ using different model chemistries.

\begin{tabular}{|c|c|c|c|c|c|c|}
\hline & $v(\mathrm{P}-\mathrm{N}) / \mathrm{cm}^{-1}$ & $\mathrm{P}-\mathrm{N} / \AA$ & $\mathrm{P}-\mathrm{Cl} / \AA \AA$ & $\mathrm{P}-\mathrm{N}-\mathrm{P} /{ }^{\circ}$ & $\mathrm{N}-\mathrm{P}-\mathrm{N} /{ }^{\circ}$ & $\mathrm{Cl}-\mathrm{P}-\mathrm{Cl} /{ }^{\circ}$ \\
\hline Experiment $^{1}$ & 1218 & 1.575 & 1.985 & 121.4 & 118.4 & 101.4 \\
\hline \multicolumn{7}{|l|}{ HF } \\
\hline CEP-31G*, 6-31G(d) ${ }^{\mathrm{a}}$ & 1286 & 1.591 & 2.027 & 121.9 & 118.2 & 102.0 \\
\hline 6-311G(d) & 1344 & 1.570 & 2.004 & 124.9 & 115.1 & 102.7 \\
\hline $6-311+G(2 d)$ & 1333 & 1.564 & 2.013 & 122.7 & 117.4 & 101.9 \\
\hline \multicolumn{7}{|l|}{ MP2 $2^{\mathrm{b}}$} \\
\hline CEP-31G*, 6-31G(d) ${ }^{\mathrm{a}}$ & 1182 & 1.620 & 2.04 & 118.8 & 121.2 & 101.9 \\
\hline $6-311 G(d)$ & 1269 & 1.590 & 2.008 & 122.8 & 117.2 & 102.8 \\
\hline $6-311+G(2 d)^{c}$ & - & 1.588 & 2.025 & 120.7 & 119.3 & 101.8 \\
\hline \multicolumn{7}{|l|}{ B3LYP $^{2, \mathrm{~d}}$} \\
\hline CEP-31G*, 6-31G(d) ${ }^{\mathrm{a}}$ & 1168 & 1.616 & 2.064 & 120.1 & 119.9 & 101.7 \\
\hline 6-311G(d) & 1225 & 1.595 & 2.038 & 123.2 & 116.8 & 102.3 \\
\hline $6-311+G(2 d)$ & 1215 & 1.589 & 2.040 & 121.3 & 118.7 & 101.6 \\
\hline \multicolumn{7}{|l|}{$\mathbf{B L Y P}^{2 \mathrm{a}, 3, \mathrm{~d}}$} \\
\hline CEP-31G*, 6-31G(d) ${ }^{a}$ & 1096 & 1.634 & 2.093 & 119.1 & 121.0 & 101.4 \\
\hline 6-311G(d) & 1149 & 1.614 & 2.070 & 122.1 & 117.9 & 102.0 \\
\hline $6-311+G(2 d)$ & 1142 & 1.608 & 2.068 & 120.4 & 119.6 & 101.3 \\
\hline $\begin{array}{l}{ }^{\mathrm{a}} \text { CEP-31G Stevens/ } \\
\text { and pseudo potential } \\
\text { numerically. }{ }^{\mathrm{c}} \text { Frequ } \\
302 \text { angular points. }\end{array}$ & $\begin{array}{l}/ \text { Krauss I } \\
\text { P and Cl, } \\
\text { s not con } \\
\text { e B3LYI }\end{array}$ & split va & nce basi & $\begin{array}{l}\mathrm{et}^{4} \text { with } \\
\text { encies }\end{array}$ & $\begin{array}{l}\text { arisatic } \\
\text { ulated } \\
75 \text { radic }\end{array}$ & $\begin{array}{l}\text { nction } \\
\text { alls and } \\
\text { radial }\end{array}$ \\
\hline
\end{tabular}


Table S-2: Optimised geometries.

\begin{tabular}{|c|c|c|c|c|}
\hline \multirow{2}{*}{$\frac{\text { Molecule }}{\left[\mathrm{H}_{2} \mathrm{NPH}_{2}\right]}$} & \multirow{2}{*}{$\mathrm{Z}$} & \multicolumn{3}{|c|}{ Coordinates } \\
\hline & & $-1 \quad 113067$ & 0.040720 & 0 \\
\hline & 15 & 0.602370 & -0.124272 & -0.022725 \\
\hline & 1 & -1.603266 & -0.802058 & $\odot .172515$ \\
\hline & 1 & 0.946419 & 1.007374 & -0.802421 \\
\hline & 1 & $\odot .952903$ & 0.528087 & 1.196249 \\
\hline & 1 & -1.540144 & 0.845581 & 0.350131 \\
\hline$\left[\mathrm{HNPH}_{3}\right]$ & & & & \\
\hline & $\begin{array}{r}15 \\
1\end{array}$ & $\begin{array}{l}0.423974 \\
1.122133\end{array}$ & $\begin{array}{l}-0.010500 \\
-0.629413\end{array}$ & $\begin{array}{l}0.000000 \\
1.074733\end{array}$ \\
\hline & 1 & 1.122133 & -0.629416 & -1.074731 \\
\hline & 1 & 0.997534 & 1.264905 & -0.000002 \\
\hline & 7 & -1.133952 & 0.126603 & $\odot . ๑ \odot \odot \odot \odot \odot ~$ \\
\hline & 1 & -1.663739 & -0.734788 & $\odot . \odot \odot \odot \odot \odot \odot$ \\
\hline$[\mathrm{H}$ & & & & \\
\hline & 7 & ๑.019296 & 1.047591 & $\odot . ๑ \odot \odot \odot \odot \odot ~$ \\
\hline & 15 & ๑.019296 & -0.532994 & 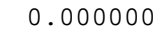 \\
\hline & 1 & -1.399390 & -0.743114 & $\odot . ๑ \odot \odot ० \odot \odot$ \\
\hline & 1 & ๑.974881 & 1.404882 & $\odot . \odot \odot \odot \odot \odot \odot$ \\
\hline$[\mathrm{H}$ & 15 & & 0 & 8 \\
\hline & $\begin{array}{r}15 \\
7\end{array}$ & $\begin{array}{l}-0.044918 \\
-0.314263\end{array}$ & $\begin{array}{r}0.0000000 \\
-0.000001\end{array}$ & $\begin{array}{l}0.446418 \\
1.946283\end{array}$ \\
\hline & 1 & 0.453977 & $-\odot .000001$ & 2.602362 \\
\hline & 17 & 0.975937 & 1.588277 & $-0.40207 \odot$ \\
\hline & 17 & 0.975945 & -1.588272 & $-0.4 \odot 207 \odot$ \\
\hline & 17 & -1.809551 & $-0.0000 \odot 4$ & -0.544248 \\
\hline $\mathrm{C}_{6} \mathrm{H}_{6}$ & 6 & 1 & ค९ & 5 \\
\hline & 6 & $\begin{array}{l}-1.205534 \\
-1.205534\end{array}$ & $\odot .000000$ & $\begin{array}{r}-0.096015 \\
0.696015\end{array}$ \\
\hline & 6 & $\odot . ๑ \odot \odot \odot \odot \odot$ & $\odot .0000 \odot \odot$ & 1.392030 \\
\hline & 6 & 1.205534 & $\odot .00000 \odot$ & $\odot .696015$ \\
\hline & 6 & 1.205534 & $\odot .00 \odot \odot \odot \odot$ & -0.696015 \\
\hline & 6 & ๑.๑००००० & $\odot .00 \odot \odot \odot \odot$ & -1.392030 \\
\hline & 1 & -2.142245 & $\odot .0000 \odot \odot$ & -1.236826 \\
\hline & 1 & -2.142245 & $\odot .0 \odot \odot \odot \odot \odot$ & 1.236826 \\
\hline & 1 & $\odot . \odot \odot \odot ० \odot \odot$ & $\odot . \odot ० \odot \odot \odot \odot$ & 2.473652 \\
\hline & 1 & 2.142245 & 0.000000 & 1.236826 \\
\hline 0 & 1 & 2.142245 & $\odot . \diamond 0000 \odot$ & -1.236826 \\
\hline & 1 & $\odot .0000 \odot \odot$ & $\odot .000000$ & -2.473652 \\
\hline
\end{tabular}




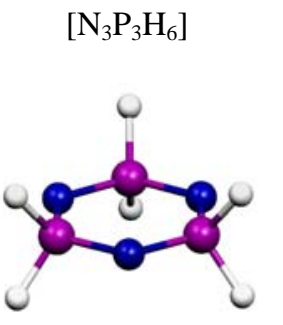

$$
\begin{array}{r}
-0.807855 \\
-0.807855 \\
1.615710 \\
0.797719 \\
0.797719 \\
-1.595437 \\
-1.254960 \\
-1.254960 \\
2.509920 \\
-1.254960 \\
-1.254960 \\
2.509920
\end{array}
$$

$\odot .0000 \odot \odot$

$\odot .000 \odot \odot \odot$

○. 000000

$\odot .000000$

๑. $00000 \odot$

$\odot .000000$

1.085282

1. 085282

- 1.085282

1. 085282

1.085282

$\left[\mathrm{N}_{3} \mathrm{P}_{3} \mathrm{~F}_{6}\right]$

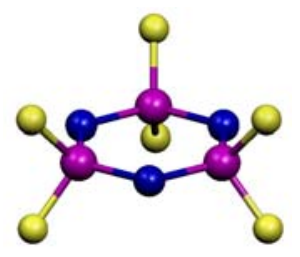

0.000000

1. 370600

$-1.370600$

0.000000

$-1.359780$

1. 359780

0.000000

2. 250060

$-2.250060$

0.000000

2. 250060

$-2.250060$

1. 085282

$\left[\mathrm{N}_{3} \mathrm{P}_{3} \mathrm{Cl}_{6}\right]$

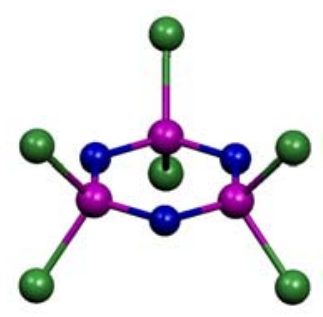

$-0.799520$

$-0.799520$

1.599040

0.789360

0.789360

$-1.578710$

$-1.444330$

$-1.444330$

2.888650

$-1.444330$

$-1.444330$

2.888650

$-1.582630$

0.791320

๑. 791320

1.570140

$-\odot .785070$

$-0.785070$

$-2.598140$

1. 299070

1. 299070

$-2.598140$

1. 299070

1. 299070

1. 384810

$-1.384810$

0.000000

$-1.367210$

1. 367210

0.000000

2. 501640

$-2.501640$

๑. 000000

2. 501640

$-2.501640$

$\odot .000000$

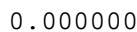

$-1.604130$

1. 389220

0.802070

๑. 802070

1.581680

0. $00 \odot \odot \circ \circ$

$-0.790840$

$-1.369780$

1. 369780

0.000000

$-0.790840$

$-2.999860$

2. 597960

1. 499930

$-2.597960$

1.499930

- 2.999860

1. 499930

$-1.399246$

1. 399246

0.000000

1. 381689

$-1.381689$

0.000000

$-2.173655$

2.173655

0.000000

$-2.173655$

2. 173655

$\odot .000000$

0.000000

○. 000000

0.000000

๑. 000000

○. 000000

๑ . $00000 \odot$

1.172920

1.172920

$-1.172920$

$-1.172920$

$-1.172920$

1.172920

0.000000

$\odot .00000 \odot$

0.000000

○. 000000

0.000000

0.000000

1. 580250

1.580250

$-1.580250$

$-1.580250$

$-1.580250$

1.580250

0.000000

$\odot .00000 \odot$

$\odot .000 \odot ० \odot$

0.000000

๑ . $0 \odot \odot \odot ० \odot$

๑. 000000

1.734680

1.734680

$-1.734680$

$-1.734680$

$-1.734680$

$\left[\mathrm{N}_{3} \mathrm{P}_{3}\left(\mathrm{CH}_{3}\right)_{6}\right]$

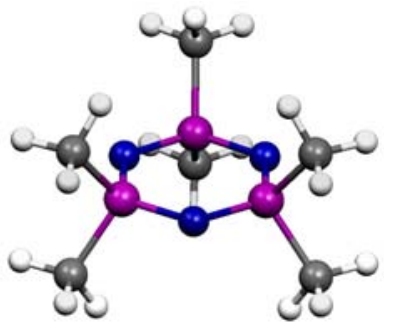

$\odot .744420$

2. 669210

$-1.422030$

.739360

2. 646600

$1.43520 \odot$

$-2.688610$

$-2.657500$

$-0.686880$

$-1.419850$

$-\odot .686540$

1.437640

1.943030

$-1.980410$

$-1.420540$

920910

1.961950

1.435350

$\odot .566320$

2.109790

$-2.340400$

.066570

3. 523440

$-1.386760$

778300

3. 017200

1.408890

0.552060

2.073650

2.343430

1.775030

2. 989280

1.434790

$\odot .066100$

3. 504810

1.409490

$-2.123490$

- 3.068410

$-3.520850$

$-0.532200$

$-2.339080$

$-1.709390$

$-1.400820$

$-2.072560$

$\odot .017800$

$-1.388850$

2. 345020 
$\left[\mathrm{N}_{3} \mathrm{P}_{3}\left(\mathrm{CF}_{6}\right)_{6}\right]$
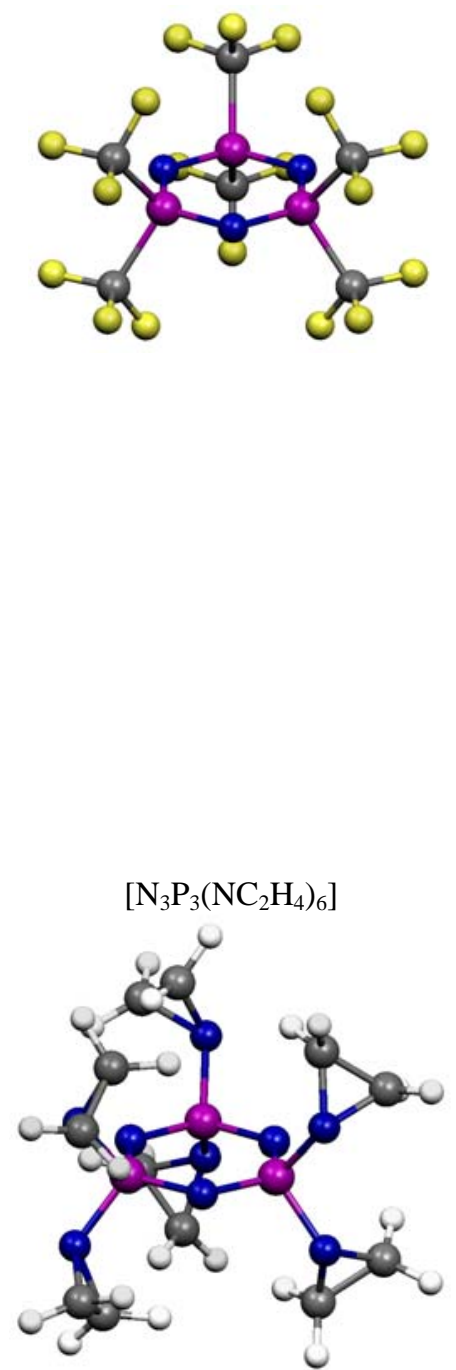

\begin{abstract}
$-3.488710$
$-3.039970$

1.527360

3. 017900

1. 752620

1.487590

1.736990

2.994900

1. 540860

$-1.113050$

$-0.428450$

0.438460

$-1.580220$

1. 140900
\end{abstract}

$-1.593270$

๑. 851720

๑. 742020

1.590270

$-0.850180$

$-0.740110$

$-2.755860$

1. 471220

1. 284690

$-2.755860$

1. 471210

1. 284680

2. 613510

๑. 939990

0.684640

2. 613500

๑. 684640

0.939980

$-3.489330$

$-2.012000$

$-3.586200$

- 3.489320

$-2.011990$

$-3.586200$

1.077600

๑. 961400

2. 810060

1. 077630

๑. $96140 \circ$

2. 810070

$-0.258339$

1. 218734

$-0.394818$

$-0.396896$

$-0.876578$

$-1.715675$

$-0.750317$

$-1.704914$

$-2.579646$

$-1.006723$

$-0.169792$

$-1.211885$

$-1.829192$

$-2.594241$

$-1.782997$

$-0.325964$

$-1.474408$

1. 522812

$-1.262169$

0.259374

2. 558702

2. 508399

$-2.153070$

$-2.122264$

2. 349224
๑. 019950

$-1.708140$

$-1.569870$

$-1.794850$

$-3.054190$

$-1.542840$

$-3.037150$

$-1.770510$

0.395630

1.136030

$-1.532010$

1.570750

$-0.405890$

$-1.164820$

๑. $00 \odot \odot \odot \odot$

๑. . $0000 \bigcirc$

0.000000

0.000000

๑. .०००००

๑. 000010

1.485810

1. 485510

$-1.484450$

$-1.485810$

$-1.485510$

1.484450

$-1.490330$

$-2.606320$

$-1.490980$

1. 490330

1. 490970

2.606320

1. 494900

2.605810

1. 493320

$-1.494890$

$-2.605810$

$-1.493330$

$-2.605510$

$-1.494470$

$-1.492590$

2. 605520

1. 494460

1. 492570

1. 608278

1. 001330

2. 720926

2. 673981

2. 201678

3. 109117

2. 266820

3. 084051

2. 568940

1. 217967

2. 715581

1. 169621

4.142703

2.668294

4.166485

2.585229

0.553692

$-0.579643$

$-1.028579$

$-1.555540$

$-1.018892$

$-0.992933$

$-1.707161$

$-1.672802$

$-0.489514$
1. 426540

1. 425170

$-2.340810$

$-1.399300$

$-1.389960$

2. 343640

1. 415040

1. 430500

$-0.009900$

$-0.011680$

$-0.019570$

- 0.003000

$-0.003540$

$-0.004480$

๑. 062580

1. 347690

$-1.412250$

$-0.063660$

$-1.347020$

1. 408120

0.110950

2. 332840

$-2.443870$

๑. 110960

2. 332830

$-2.443870$

$-2.629760$

$-1.787260$

$-3.643830$

$-2.629770$

$-3.643830$

$-1.787260$

1. 234430

๑. 077310

$-0.942860$

1. 234450

0.077320

$-0.942840$

1. 700760

3. 573870

2. 419800

1. 700770

3.573880

2. 419820

- 0.001770

- 0.017218

$-1.263136$

1. 300150

2. 599664

1.775003

$-2.607502$

$-1.814403$

$-1.425016$

- 2.742315

$-3.409015$

2.676660

$-2.025829$

1. 310441

2. 015463

3. 454524

$-0.024526$

๑. 006707

$-0.000074$

$-0.011579$

$-1.250867$

1. 313141

$-1.262123$

1. 301582

$-2.598001$ 


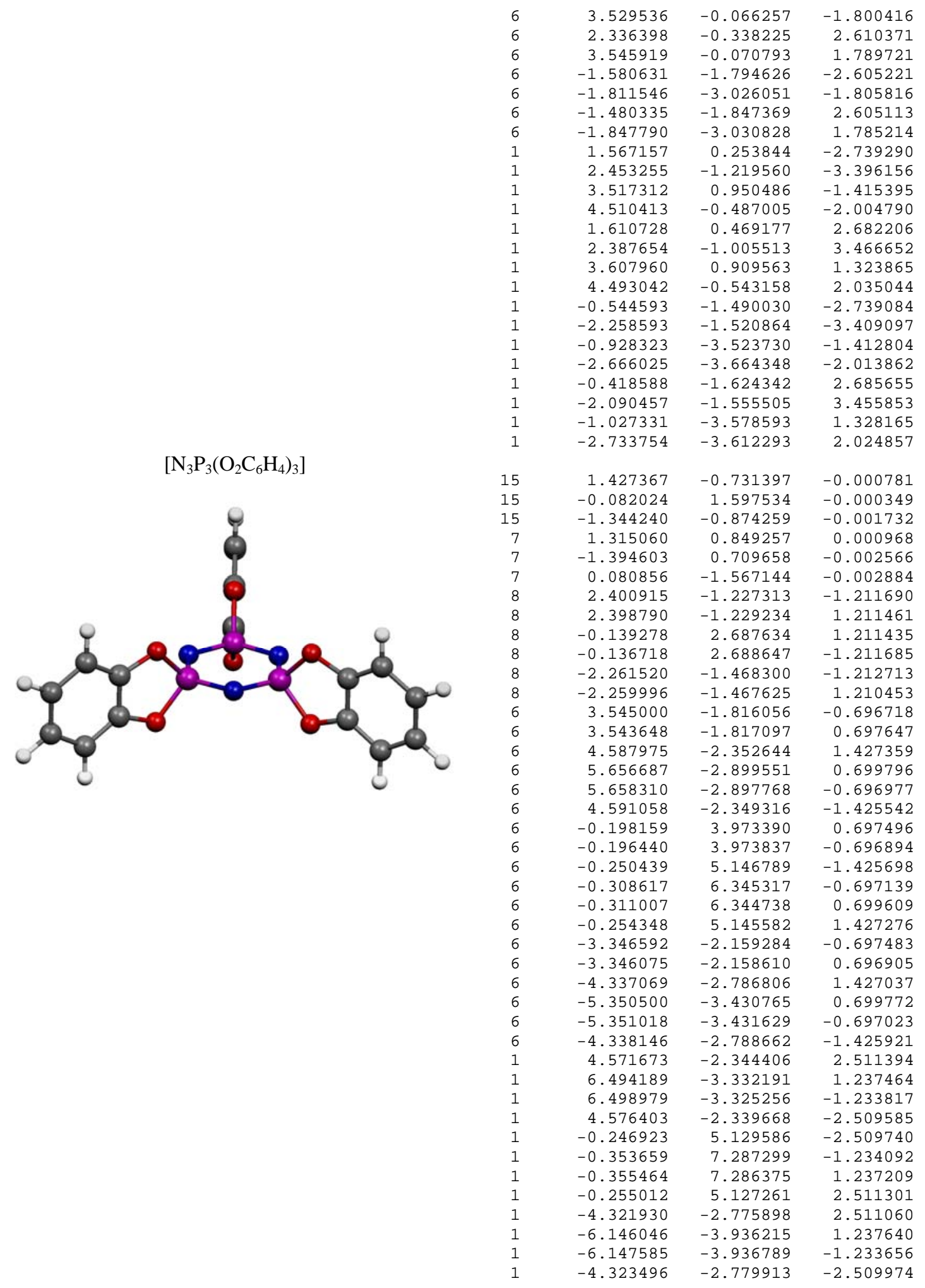




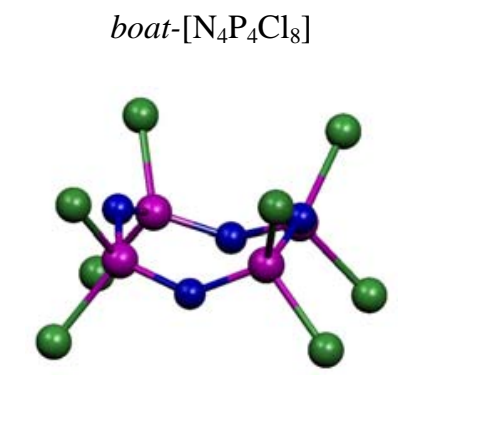

-1.303975
-1.794304
-2.474050
-1.773465
0.178204
-1.574359
1.574363
-0.178203
-2.191874
-2.909701
1.794317
2.191887
2.909697
1.303975
2.474047
1.773450

$-1.574361$

$-0.178198$

0.026482

$-2.191810 \quad 1.582768$

$-2.909713-1.445405$

$-1.794357 \quad 0.519780$

$1.303977-0.026501$

$-1.303982-0.026496$

$1.794346 \quad 0.519792$

$2.474060 \quad-1.582753$

$1.773396 \quad 1.445420$

$0.178198-0.519805$

$-2.474049-1.582758$

$-1.773421 \quad 1.445425$

1.574362

$2.191843 \quad 1.582765$

$2.909703-1.445414$

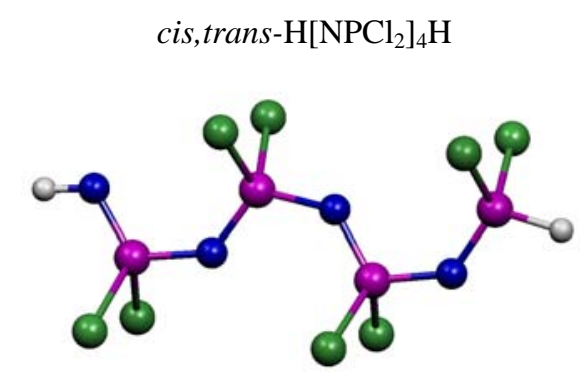

-3.015617
-1.482764
-0.620794
-0.609380
-0.678224
-1.178408
-2.368797
-2.373275
0.000246
1.550239
2.317299
2.315152
2.321606
1.777151
0.681023
0.677476
-3.555108
2.847764

$-2.997826$

$-3.115553$

$-4.173696$

$-4.181964$

$-1.713844$

$-0.245359$

๑. 266062

๑. 268961

0.852616

$\odot .702298$

$-0.319677$

$-0.317974$

2. 123689

3.571844

4.164223

4.166804

$-3.855529$

4.468084

$-\odot .0 \odot 6362$
-0.000866
1.591719
-1.581434
$-0.0 \odot 2414$
$-0.0 \odot \odot 5 \odot 8$
1.593243
-1.589784
-0.001334
$-0.0 \odot 2422$
1.587866
-1.594813
-0.002243
$-0.00 \odot 487$
1.606469
$-1.6 \odot 4 \odot 49$
$-0.0 \odot 5015$
$-0.0 \odot \odot 95 \odot$

847764

\section{S2. Topological properties}

For the topological analysis of the electron density, as described by Bader's AIM theory, ${ }^{5}$ it is sufficient to note that our calculations using density functional theory, B3LYP, and larger basis sets [typically 6-311+G(2d)] essentially reproduce those of Luaña and co-workers, ${ }^{6}$ carried out at the HF/6-31G** level. Our results are summarised in Table S-3, Tables 2, 3 and 6 in the main text, and selected critical points of $\left[\mathrm{N}_{3} \mathrm{P}_{3} \mathrm{Cl}_{6}\right]$ are depicted in Figure S-1. Relevant discussion is also included in the main text, although in addition to the analysis of Luaña and co-workers we note that:

- Localised concentrations of electron density $\left(\nabla^{2} \rho<0\right)$ ca. $0.4 \AA$ from $\mathrm{N}$ centres, (3, 3) critical points, can be located. Such concentrations of electron density resemble, at least in valence bond terms, lone pairs of electrons. An illustration of this electron density is depicted in Figure S-2 (created using MORHY98 ${ }^{7}$ ). 
- Amongst the phosphazenes, there is little variation in the distance, $r_{\mathrm{N}}$, from the $(3,-3)$ critical point to the $\mathrm{N}$ centre, although variations in both the electron density, $\rho$, and the Laplacian of the electron density, $\nabla^{2} \rho$, correlate well with the occupations of the $\mathrm{N}_{\mathrm{lp} 1}$ and $\mathrm{N}_{\mathrm{lp} 2} \mathrm{NBOs}$, reinforcing the idea of negative hyperconjugation ( $c f . Q(\mathrm{~N})$ remains almost constant for all the phosphazenes).

- In the context of negative hyperconjugation in these molecules, discussion of bond ellipticity values (characteristic of the symmetry about a bond) is difficult because both in-plane and out-of plane $\pi$-type bonding is possible. For the model compounds, however, a clear trend is evident, with the phosphazene like $\left[\mathrm{HNPH}_{3}\right]$ having a $\mathrm{P}-\mathrm{N}$ bond ellipticity of 0.165 much closer to that of [NHPH] with a formal double bond ( $\varepsilon$ $=0.233$ ) than $\left[\mathrm{H}_{2} \mathrm{NPH}_{2}\right]$ that has a value close to zero, as expected for a cylindrically symmetric $\sigma$-type bonding. These results agree well with the conclusions of the NRT theory; that there is significant multiple bond character to the P-N bond (see section S3 below) and by inference the phosphazene P-N bond as well.

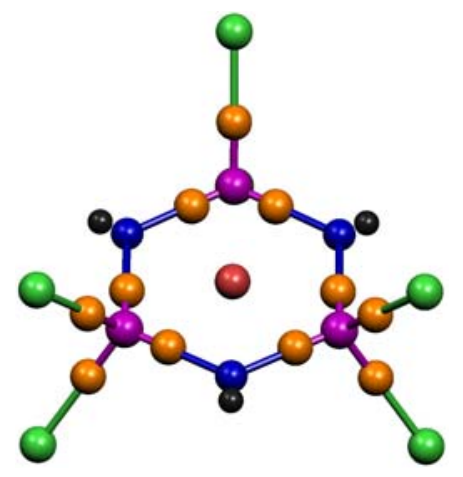

Figure S-1: Location of the $(3,-1)$ [orange], $(3,+1)$ [red], and selected $(3,-1)$ [black] critical points in $\left[\mathrm{N}_{3} \mathrm{P}_{3} \mathrm{Cl}_{6}\right]$.

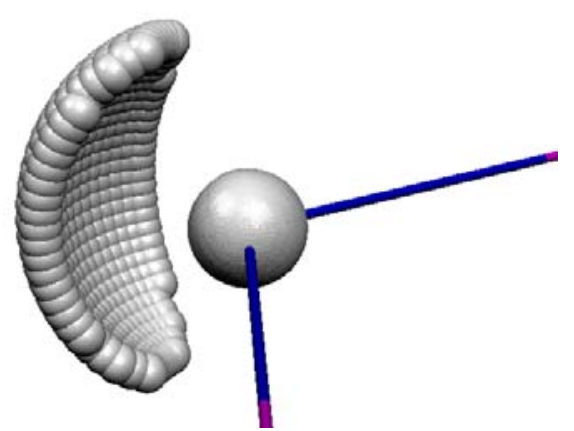

Figure S-2: $\nabla^{2} \rho$ isosurface $\left(-1.5\right.$ e $\left.\AA^{-5}\right)$ near $\mathrm{N}$ in $\left[\mathrm{N}_{3} \mathrm{P}_{3} \mathrm{Cl}_{6}\right]$. 
Table S-3: Selected critical point properties. ${ }^{a}$

\begin{tabular}{|c|c|c|c|c|c|c|c|c|c|c|c|c|}
\hline & \multicolumn{4}{|c|}{ P-N bond $(3,-1)$} & \multicolumn{3}{|c|}{$\mathrm{N}$ "lone pair” $(3,-3)$} & \multicolumn{3}{|c|}{ P-X bond $(3,-1)$} & \multicolumn{2}{|c|}{ Ring $(3,+1)$} \\
\hline & $r_{\mathrm{N}}[\AA] / r_{\mathrm{P}}[\AA]$ & $\rho_{3}^{\rho / \mathrm{e}^{-}}$ & $\nabla^{2} \rho / \mathrm{e}^{-}$ & $\varepsilon$ & $r_{\mathrm{N}} / \AA$ & $\rho / \mathrm{e} \AA^{-3}$ & $\nabla^{2} \rho_{5} / \mathrm{e}^{-}$ & $\rho / \mathrm{e} \AA^{-3}$ & $\nabla^{2} \rho / \mathrm{e}^{-}$ & $\varepsilon$ & $\rho / \mathrm{e}_{3}^{-}$ & $\nabla^{2} \rho / \mathrm{e} \AA$ \\
\hline$\left[\mathrm{H}_{2} \mathrm{NPH}_{2}\right]$ & $1.040 / 0.686$ & 0.161 & 0.080 & 0.013 & 0.398 & 0.544 & -2.266 & 0.165 & -0.263 & 0.101 & & \\
\hline$\left[\mathrm{HNPH}_{3}\right]$ & $0.932 / 0.634$ & 0.219 & 0.417 & 0.165 & $0.406^{\mathrm{C}}$ & $0.488^{\mathrm{C}}$ & $-1.752^{\mathrm{C}}$ & $\begin{array}{l}0.170^{\mathrm{d}} \\
0.178^{\mathrm{e}}\end{array}$ & $\begin{array}{l}-0.330^{\mathrm{d}} \\
-0.313^{\mathrm{e}}\end{array}$ & $\begin{array}{l}0.053^{\mathrm{d}} \\
0.076^{\mathrm{e}}\end{array}$ & & \\
\hline [HNPH] & $0.949 / 0.632$ & 0.211 & 0.516 & 0.233 & 0.392 & 0.557 & -2.516 & 0.166 & -0.327 & 0.010 & & \\
\hline$\left[\mathrm{N}_{3} \mathrm{P}_{3} \mathrm{H}_{6}\right]$ & $0.961 / 0.645$ & 0.202 & 0.281 & 0.093 & 0.407 & 0.480 & -1.641 & 0.180 & -0.370 & 0.038 & 0.022 & 0.088 \\
\hline$\left[\mathrm{N}_{3} \mathrm{P}_{3} \mathrm{~F}_{6}\right]$ & $0.942 / 0.635$ & 0.219 & 0.324 & 0.067 & 0.405 & 0.488 & -1.705 & 0.184 & 0.777 & 0.011 & 0.025 & 0.097 \\
\hline$\left[\mathrm{N}_{3} \mathrm{P}_{3} \mathrm{Cl}_{6}\right]$ & $0.950 / 0.640$ & 0.210 & 0.317 & 0.088 & 0.404 & 0.494 & -1.772 & 0.134 & -0.202 & 0.060 & 0.023 & 0.094 \\
\hline$\left[\mathrm{N}_{3} \mathrm{P}_{3} \mathrm{Br}_{6}\right]^{\mathrm{b}}$ & $0.971 / 0.623$ & 0.197 & 0.807 & 0.105 & 0.398 & 0.505 & -2.070 & 0.119 & -0.152 & 0.045 & 0.019 & 0.095 \\
\hline$\left[\mathrm{N}_{3} \mathrm{P}_{3}\left(\mathrm{CH}_{3}\right)_{6}\right]$ & $0.966 / 0.646$ & 0.201 & 0.265 & 0.082 & 0.407 & 0.480 & -1.640 & 0.171 & -0.400 & 0.037 & 0.021 & 0.087 \\
\hline$\left[\mathrm{N}_{3} \mathrm{P}_{3}\left(\mathrm{CF}_{3}\right)_{6}\right]^{\mathrm{b}}$ & $0.971 / 0.623$ & 0.197 & 0.816 & 0.101 & 0.400 & 0.495 & -1.944 & 0.166 & -0.261 & 0.058 & 0.020 & 0.095 \\
\hline$\left[\mathrm{N}_{3} \mathrm{P}_{3}\left(\mathrm{NC}_{2} \mathrm{H}_{4}\right)_{6}\right]^{\mathrm{b}}$ & $\begin{array}{l}0.984 / 0.627^{s} \\
0.974 / 0.623^{1}\end{array}$ & $\begin{array}{l}0.198^{\mathrm{S}} \\
0.193^{\mathrm{l}}\end{array}$ & $\begin{array}{l}0.787^{\mathrm{s}} \\
0.747^{1}\end{array}$ & $\begin{array}{l}0.080^{\mathrm{s}} \\
0.082^{1}\end{array}$ & 0.400 & 0.497 & -1.965 & 0.175 & 0.433 & 0.037 & 0.019 & 0.092 \\
\hline$\left[\mathrm{N}_{3} \mathrm{P}_{3}\left(\mathrm{O}_{2} \mathrm{C}_{6} \mathrm{H}_{4}\right)_{3}\right]^{\mathrm{b}}$ & $0.966 / 0.620$ & 0.203 & 0.833 & 0.080 & 0.398 & 0.503 & -2.037 & 0.169 & 0.703 & 0.030 & 0.020 & 0.099 \\
\hline$\left[\mathrm{N}_{4} \mathrm{P}_{4} \mathrm{Cl}_{8}\right]$ & $0.942 / 0.636$ & 0.212 & 0.368 & 0.052 & 0.406 & 0.490 & -1.698 & 0.134 & -0.202 & 0.063 & 0.006 & 0.031 \\
\hline $\mathrm{H}\left[\mathrm{NPCl}_{2}\right]_{4} \mathrm{H}^{\mathrm{f}}$ & $\begin{array}{l}0.926 / 0.630^{\mathrm{s}} \\
0.968 / 0.647^{1}\end{array}$ & $\begin{array}{l}0.222^{\mathrm{s}} \\
0.200^{\mathrm{l}}\end{array}$ & $\begin{array}{l}0.455^{\mathrm{s}} \\
0.230^{\mathrm{l}}\end{array}$ & $\begin{array}{l}0.089^{s} \\
0.081^{1}\end{array}$ & 0.406 & 0.487 & -1.673 & 0.128 & -0.175 & 0.030 & & \\
\hline
\end{tabular}

${ }^{\mathrm{a}}$ Values are averaged. $\mathrm{X}$ refers to the substituent atom bound directly to $\mathrm{P} .{ }^{\mathrm{b}}$ Using the 6-31G(d,p) basis set for all atoms to generate the AIMPAC input file. ${ }^{\mathrm{c}}$ Two $(3,-3)$ critical points are found, $\pm 0.57 \AA$ out of the $\mathrm{H}-\mathrm{N}-\mathrm{P}-\mathrm{H}^{\mathrm{e}}$ plane. ${ }^{\mathrm{d}} \mathrm{H}$ atoms syn to the $\mathrm{N}-\mathrm{H}$ bond. ${ }^{\mathrm{e}} \mathrm{H}$ atom anti to the $\mathrm{N}-\mathrm{H}$ bond. ${ }^{\mathrm{f}}$ Terminal $\mathrm{NH}$ and $\mathrm{PCl}_{2} \mathrm{H}$ groups excluded from the average. ${ }^{\mathrm{s}}$ Quantity across the shorter $\mathrm{P}-\mathrm{N}$ bond. ${ }^{1}$ Quantity across the longer P-N bond. 


\section{S3. NRT analysis of $\left[\mathrm{H}_{2} \mathrm{NPH}_{2}\right],\left[\mathrm{HNPH}_{3}\right]$, and [HNPH]}

The results from the Natural Resonance Theory (NRT) analysis are schematically represented in Figure 2 (main text), with NRT bond orders given in Table S-4. Both $\left[\mathrm{H}_{2} \mathrm{NPH}_{2}\right]$ and [HNPH] have dominant Lewis structures, 94.8\% and 97.2\%, containing formally single and double P-N bonds, respectively, as expected. The P-N NRT bond orders indicate that while these bonds are covalent, they are also significantly polarised. Correspondingly, the $\sigma_{\mathrm{PN}}$ and $\pi_{\mathrm{PN}}$ NBOs of the leading resonance structures are largely $\mathrm{N}$ centred; $\sigma_{\mathrm{PN}}\left(\left[\mathrm{H}_{2} \mathrm{NPH}_{2}\right]\right), 71.7 \% ; \sigma_{\mathrm{PN}}([\mathrm{HNPH}]), 64.2 \% ; \pi_{\mathrm{PN}}([\mathrm{HNPH}]), 70.6 \%$, see Figure S-3 for illustrations.

Table S-4: NRT bond orders.

\begin{tabular}{cccccc}
\hline & $\mathrm{N}-\mathrm{H}$ & $\mathrm{N}$ & $\mathrm{N}-\mathrm{P}$ & $\mathrm{P}$ & $\mathrm{P}-\mathrm{H}$ \\
\hline$\left[\mathrm{H}_{2} \mathrm{NPH}_{2}\right]$ & 0.99 & 0.97 & 1.04 & 1.00 & 0.98 \\
covalent & 0.61 & - & 0.56 & - & 0.92 \\
ionic & 0.38 & - & 0.47 & - & 0.06 \\
[HNPH $\left._{3}\right]$ & 0.99 & 1.83 & 1.19 & 0.01 & $0.92^{\mathrm{a}}, 0.96^{\mathrm{b}}$ \\
covalent & 0.62 & - & 0.71 & - & $0.89^{\mathrm{a}}, 0.94^{\mathrm{b}}$ \\
ionic & 0.36 & - & 0.48 & - & $0.02^{\mathrm{a}}, 0.02^{\mathrm{b}}$ \\
[HNPH $]^{\mathrm{H}}$ & 0.99 & 0.99 & 2.02 & 1.00 & 0.97 \\
covalent & 0.64 & - & 1.30 & - & 0.88 \\
ionic & 0.35 & - & 0.72 & - & 0.10 \\
\hline
\end{tabular}

${ }^{\mathrm{a}} \mathrm{H}$ atoms syn to the $\mathrm{N}-\mathrm{H}$ bond. ${ }^{\mathrm{b}} \mathrm{H}$ atom anti to the $\mathrm{N}-\mathrm{H}$ bond.

The zwitterionic-like Lewis structure is the most dominant resonance structure for $\left[\mathrm{HNPH}_{3}\right], 79.2 \%$. The most important secondary contributions are Lewis forms with a P$\mathrm{N}$ double bond, corresponding to complete negative hyperconjugation from a $\mathrm{N}_{\mathrm{lp}} \mathrm{NBO}$ to either of the syn (to the N-H bond) $\sigma_{\text {PH }}^{*}$ NBOs, as evidenced by a large donor-acceptor interaction between these NBOs in the second order perturbation analysis (18.78 kcal $\mathrm{mol}^{-1}$ ), see Figure S-3. The P-N NRT bond order is slightly larger than that in $\left[\mathrm{H}_{2} \mathrm{NPH}_{2}\right]$ owing to a larger covalent contribution, presumably as a result of the negative hyperconjugation. Like the other molecules, the dominant resonance structure of $\left[\mathrm{HNPH}_{3}\right]$ has a $\mathrm{N}$ centered $\sigma_{\mathrm{PN}} \mathrm{NBO}(67.2 \%)$. Owing to the necessity to correct for negative hyperconjugation the N lone pair NBOs deviate from their ideal occupancy of 
$2.0(1.75,1.73)$ and there is significant occupancy of the $\sigma_{\mathrm{PH}}^{*}$ NBOs $\left(0.15\right.$ per syn $\sigma_{\mathrm{PH}}^{*}$, 0.07 per anti $\left.\sigma_{\mathrm{PH}}^{*}\right)$ and $\sigma_{\mathrm{PN}}^{*} \mathrm{NBO}(0.07)$

Together the zwitterionic and negative hyperconjugation resonance forms represent a suitable description of the bonding in $\left[\mathrm{HNPH}_{3}\right]$ with a combined weighting of $94.8 \%$; the overall importance of the negative hyperconjugation is $16 \%$.

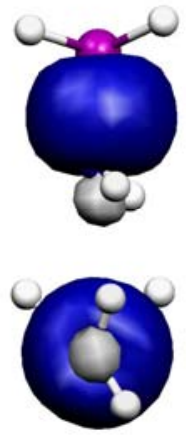

$\sigma_{\mathrm{PN}}$
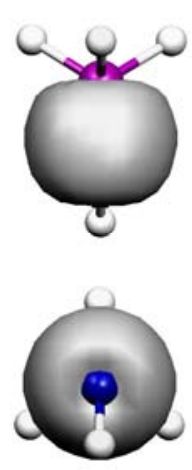

$\sigma_{\mathrm{PN}}$
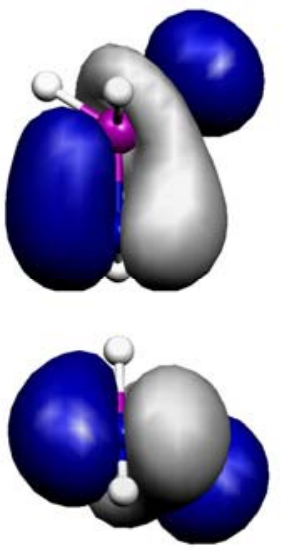

$\mathbf{N}_{\text {lp }} \rightarrow \sigma_{\text {PH }}^{*}$ (18.78 kcal mol$^{-1}$ )
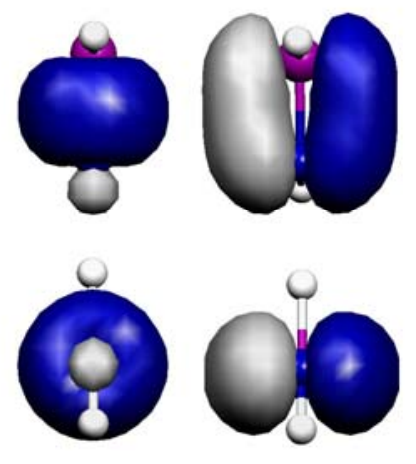

$\sigma_{\mathrm{PN}}$ and $\pi_{\mathrm{PN}}$

Figure S-3: Selected P-N NBOs of the leading resonance structures. 
S4. NCS Analysis

Table S-5: Major NBO contributions to the NICS values as determined by NCS analysis.

\begin{tabular}{|c|c|c|c|c|c|c|c|c|c|}
\hline & \multirow[t]{2}{*}{$\operatorname{NICS}\left(d^{\mathrm{a}}\right)$} & \multicolumn{2}{|c|}{$\mathrm{N}_{\mathrm{lp} 1}$} & \multicolumn{2}{|c|}{$\mathrm{N}_{\mathrm{lp} 2}$} & \multicolumn{2}{|c|}{ PN } & \multicolumn{2}{|c|}{ PX } \\
\hline & & $\mathrm{L}$ & NL & L & NL & $\mathrm{L}$ & NL & $\mathrm{L}$ & NL \\
\hline \multirow[t]{2}{*}[\mathrm{N}_{3}\mathrm{P}_{3}\mathrm{H}_{6}]{} & $+0.04(0)$ & +4.67 & -0.34 & -0.09 & -3.53 & -0.98 & -0.20 & +1.01 & -0.46 \\
\hline & $+1.23(1)$ & +1.80 & -0.17 & +1.27 & -1.99 & -0.77 & -0.10 & $\begin{array}{l}+0.51^{\mathrm{g}} \\
+1.09^{\circ}\end{array}$ & $\begin{array}{l}-0.30^{\mathrm{g}} \\
-0.39^{\circ}\end{array}$ \\
\hline \multirow[t]{2}{*}[\mathrm{N}_{3}\mathrm{P}_{3}\mathrm{F}_{6}]{} & $-5.13(0)$ & +4.36 & -1.38 & -0.28 & -3.95 & -0.81 & -0.27 & +0.30 & +0.05 \\
\hline & $-2.45(1)$ & +1.36 & -0.56 & +1.32 & -2.31 & -0.68 & -0.17 & $\begin{array}{l}-0.14^{\mathrm{g}} \\
+0.44^{\circ}\end{array}$ & $\begin{array}{l}+0.04^{\mathrm{g}} \\
+0.03^{\mathrm{o}}\end{array}$ \\
\hline \multirow[t]{2}{*}[\mathrm{N}_{3}\mathrm{P}_{3}\mathrm{Cl}_{6}]{} & $-2.52(0)$ & +3.96 & -0.74 & -0.04 & -4.36 & +0.10 & -0.64 & +0.35 & +0.41 \\
\hline & $-0.96(1)$ & +1.52 & -0.37 & +1.15 & -2.33 & -0.26 & -0.39 & $\begin{array}{l}-0.45^{\mathrm{g}} \\
+0.86^{\circ}\end{array}$ & $\begin{array}{l}+0.60^{\mathrm{g}} \\
+0.14^{\mathrm{o}}\end{array}$ \\
\hline \multirow[t]{2}{*}[\mathrm{N}_{3}\mathrm{P}_{3}\mathrm{Br}_{6}]{} & $-1.67(0)$ & +3.99 & -0.14 & +0.09 & -4.66 & +0.21 & -0.88 & +0.17 & +0.37 \\
\hline & $-0.36(1)$ & +1.59 & -0.19 & +1.23 & -2.50 & -0.13 & -0.52 & $\begin{array}{l}-0.81^{\mathrm{g}} \\
+0.85^{\circ}\end{array}$ & $\begin{array}{l}+0.61^{\mathrm{g}} \\
+0.12^{\mathrm{o}}\end{array}$ \\
\hline \multirow[t]{2}{*}[\mathrm{N}_{3}\mathrm{P}_{3}(\mathrm{CH}_{3})_{6}]{} & $-1.91(0)$ & +4.72 & -1.75 & -0.16 & -4.04 & -0.25 & +0.05 & +0.52 & +0.12 \\
\hline & $-0.42(1)$ & +1.67 & -0.73 & +1.12 & -2.26 & -0.49 & +0.08 & $\begin{array}{l}+0.11^{\mathrm{g}} \\
+0.92^{\mathrm{o}}\end{array}$ & $\begin{array}{l}+0.23^{\mathrm{g}} \\
+0.03^{\circ}\end{array}$ \\
\hline \multirow[t]{2}{*}[\mathrm{N}_{3}\mathrm{P}_{3}(\mathrm{CF}_{3})_{6}]{$^{\mathrm{b}}$} & $-3.88(0)$ & +5.45 & -1.53 & +0.35 & -3.32 & -1.72 & +0.12 & -0.99 & +0.66 \\
\hline & $-1.92(1)$ & +1.94 & -0.53 & +1.39 & -1.93 & -1.17 & +0.10 & $\begin{array}{l}-1.53^{\mathrm{g}} \\
-0.13^{\mathrm{o}}\end{array}$ & $\begin{array}{l}+0.72^{\mathrm{g}} \\
+0.36^{\mathrm{o}}\end{array}$ \\
\hline \multirow[t]{2}{*}[\mathrm{N}_{3}\mathrm{P}_{3}(\mathrm{NC}_{2}\mathrm{H}_{4})_{6}]{$^{\mathrm{c}}$} & $-5.89(0)$ & +4.97 & -1.58 & +0.12 & -3.29 & $-1.68^{\mathrm{s}},-1.84^{\mathrm{l}}$ & $+0.14^{\mathrm{s}},-0.03^{\mathrm{l}}$ & +0.32 & +0.29 \\
\hline & $-3.71(1)$ & +1.80 & -0.65 & +1.30 & -2.03 & $-1.09^{\mathrm{s}},-1.29^{\mathrm{l}}$ & $+0.08^{\mathrm{s}}, 0$ & $\begin{array}{l}-0.04^{\mathrm{g}} \\
+0.56^{\circ}\end{array}$ & $\begin{array}{l}+0.25^{\mathrm{g}} \\
+0.15^{\mathrm{o}}\end{array}$ \\
\hline \multirow{2}{*}[\mathrm{N}_{3}\mathrm{P}_{3}(\mathrm{O}_{2}\mathrm{C}_{6}\mathrm{H}_{4})_{3}]{} & $-5.57(0)$ & +4.80 & -1.44 & -0.27 & -3.65 & -1.96 & +0.24 & +0.49 & -0.03 \\
\hline & $-2.89(1)$ & +1.61 & -0.58 & +1.10 & -2.16 & -1.32 & +0.14 & $\begin{array}{l}-0.25^{\mathrm{g}} \\
+0.81^{\mathrm{o}}\end{array}$ & $\begin{array}{l}+0.12^{\mathrm{g}} \\
-0.16^{\mathrm{o}}\end{array}$ \\
\hline
\end{tabular}


${ }^{a}$ Distance out of the plane $(\AA) .{ }^{\mathrm{g}}$ Ghost atom face. ${ }^{\circ}$ Opposing face to the ghost atom. ${ }^{\mathrm{b}}$ Using the 6-31G(d,p) basis set for the NCS calculation. ${ }^{\text {c }}$ For the NCS calculation the 6-31G(d) basis set was used for P, N and O, and 6-31G for $\mathrm{C}$ and H. ${ }^{\mathrm{s}}$ Quantity across the shorter P-N bond. ${ }^{1}$ Quantity across the longer P-N bond. 


\section{S5. References}

1. (a) Bullen, G. J.; J. Chem. Soc. A, 1971, 1450. (b) Allcock, H. R.; Chem. Rev., 1972, 72, 315.

2 (a) Becke, A. D.; Phys. Rev. A, 1988, 38, 3098. (b) Becke, A. D.; J. Chem. Phys., 1993, 98, 5648.

3 (a) Lee, C.; Yang, W.; Parr, R. G.; Phys. Rev. B, 1988, 37, 785. (b) Miehlich, B.; Savin, A.; Stoll, H.; Preuss, H.; Chem. Phys. Lett., 1989, 157, 200.

4. (a) Stevens, W.; Basch, H.; Krauss, J.; J. Chem. Phys., 1984, 81, 6026. (b) Stevens, W. J.; Krauss, M.; Basch, H.; Jasien, P. G.; Can. J. Chem., 1992, 70, 612. (c) Cundari T. R.; Stevens, W. J.; J. Chem. Phys., 1993, 98, 5555.

5. $\quad$ Leading references include: (a) Bader, R. F. W.; Atoms in Molecules - A Quantum Theory, Oxford University Press: Oxford, 1990. (b) Bader, R. F. W.; Chem. Rev., 1991, 91, 893-928. (c) Bader, R. F. W.; Encyclopedia of Computational Chemistry, eds., Schleyer, P. v. R.; Allinger, N. L.; Clark, T.; Gasteiger, J.; Kollman, O. A.; Schaefer III, H. F.; Schreiner, P. R.; John Wiley \& Sons: Chichester, Vol 1, 1998, 64-86. (d) Fradera, X.; Austen, M. A.; Bader, R. F. W.; J. Phys. Chem. A, 1999, 103, 304-314. Reference 6 also includes a excellent (and brief) introduction to the theory.

6. $\quad$ Luaña, V.; Pendás, A. M.; Costales, A.; Carriedo, G. A.; García-Alonso, F. J.; J. Phys. Chem. A, 2001, 105, 5280-5291.

7. MORPHY98, Popelier, P. L. A.; Bone, R. G. A.; UMIST, Manchester, England, 1998. 